\section{Abstracts from international Emergency Medicine journals}

Editor's note: EMJ has partnered with the journals of multiple international emergency medicine societies to share from each a highlighted research study, as selected by their editors.

Ellen J Weber

\section{African Journal of Emergency Medicine}

Official Journal of the African Federation for Emergency Medicine, the Botswana Society for Emergency Care, the Emergency Medicine Association of Tanzania, the Emergency Medicine Society of South Africa, the Ethiopian Society of Emergency Medicine Professionals and the Libyan Emergency Medicine Association

\section{Characterization of in-hospital cardiac arrest in adult patients at a tertiary hospital in Kenya}

BW Wachira, Tyler MD.

Introduction In-hospital cardiac arrest (IHCA) is defined as a cardiac arrest that occurs in a hospital and for which resuscitation is attempted. Despite the increased morbidity and mortality, IHCA incidence and outcomes remain largely unknown especially in sub-Saharan Africa. This study describes the baseline characteristics, pre-arrest physiological parameters and the rate of survival to hospital dis-

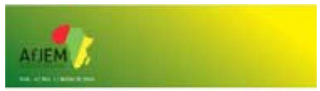
Arisan Journal of Emergency Medicine

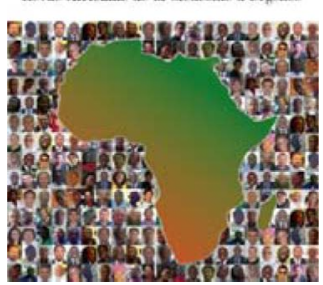
charge of adult patients with an IHCA at a tertiary hospital in Kenya.

Methods This was a retrospective chart review. Data on patient characteristics, pre-arrest physiological parameters and discharge condition were collected on all patients 18 years of age or older with an IHCA at the Aga Khan University Hospital, Nairobi, from January 2013 to December 2013.

Results The main study population comprised 108 patients. The mean age was $59.3 \pm 18.4$ years and 63 (58.3\%) patients were men. The initial rhythm post cardiac-arrest was pulseless electrical activity $(41.7 \%)$ or asystole $(35.2 \%)$ in the majority of cases. Hypertension (43.5\%), septicaemia (40.7\%), renal insufficiency (30.6\%), diabetes mellitus (25.9\%) and pneumonia $(15.7 \%)$ were the leading pre-existing conditions in the patients. A Modified Early Warning Score (MEWS) of 5 or more was reached in $56(67.5 \%, \mathrm{n}=83)$ patients before the cardiac arrest. The rate of survival to hospital discharge was $11.1 \%$. All the patients who survived to hospital discharge had a good neurological outcome.

Conclusions Early identification of warning signs that precede many in-hospital arrests may enable institution of treatment to prevent patient deterioration. Local hospitals should be encouraged to provide patients with resuscitation services and equipment in line with evidence-based programmes.

\section{Annals of Emergency Medicine}

Official Journal of the American College of Emergency Physicians

The impact of clinical decision rules on computed tomography use and yield for pulmonary embolism: A systematic review and meta-analysis

Ralph C. Wang, Stephen Bent, Ellen Weber, Jersey Neilson, Rebecca Smith-Bindman, Jahan Fahimi.

Study objective Validation studies have confirmed the accuracy of clinical decision rules for the evaluation of pretest probability of pulmonary embolism. It has been assumed that clinical decision rules will also decrease testing in actual practice, but the evidence for this is unclear. We perform a systematic review of impact analyses on clinical decision rules for pulmonary embolism.

Methods MEDLINE, EMBASE, and the Cochrane Library were searched without language restriction for studies assessing the effect of clinical decision rules on efficiency (computed tomography [CT] angiography use and yield) and safety (missed pulmonary embolism) through October 2014. Two reviewers independently extracted data on study characteristics, methods, risk of bias, and outcomes.

Results Eight studies $(n=6,677)$ contained sufficient information, including 1 randomized trial and 7 observational studies. Because of heterogeneity, the results of 4 studies of moderate to high quality assessing the Wells criteria were pooled. The pooled CT angiography yield was 9\% (95\% confidence interval [CI] $6 \%$ to $12 \%$ ) in the control group and $12 \%$ (95\% CI $11 \%$ to $14 \%$ ) in the intervention group, for a $3 \%$ increase in yield (95\% CI $1 \%$ to $5 \%$ ), relative risk 1.3 (95\% CI 1.1 to 1.6$)$. We were unable to report a pooled estimate of CT angiography use. Of 2 studies with sufficient information, there was no difference in the rate of missed pulmonary embolism between intervention and control groups. No studies used a cluster-randomized design.

Conclusion Among participants with suspected pulmonary embolism, implementation of the Wells criteria was associated with a modest increase in CT angiography yield. There is a lack of cluster-randomized trials to confirm the efficacy of clinical decision rules for the diagnosis of pulmonary embolism. 


\section{CJEM}

\section{Official journal of Canadian Association of Emergency Physicians}

\section{Hypertonic saline in severe traumatic brain injury: a systematic review and meta-analysis of randomized controlled trials}

Elyse Berger-Pelleiter, MD, MSc Marcel Émond, MD, MSc; François Lauzier, MD, MSC; Jean-François Shields, MD; Alexis F. Turgeon MD, MSc.

Objectives Hypertonic saline solutions are increasingly used to treat increased intracranial pressure following severe traumatic brain injury. However, whether hypertonic saline provides superior management of intracranial pressure and improves outcome is unclear. We thus conducted a systematic review to evaluate the effect of hypertonic saline in patients with severe trau-

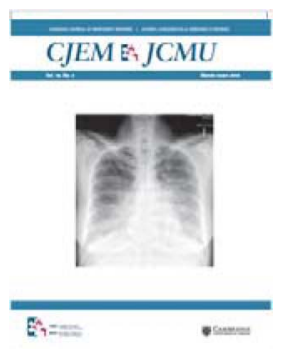
matic brain injury.

Methods Two researchers independently selected rando- mized controlled trials studying hypertonic saline in severe traumatic brain injury and collected data using a standardized abstraction form. No language restriction was applied. We searched MEDLINE, EMBASE, Cochrane Central Register of Controlled Trials, Scopus, Web of Science, and BIOSIS databases. We searched grey literature via OpenGrey and National Technical Information Service databases. We searched the references of included studies and relevant reviews for additional studies.

Results Eleven studies (1,820 patients) were included. Hypertonic saline did not decrease mortality (risk ratio 0.96 , $95 \%$ confidence interval [CI] 0.83 to $1.11, \mathrm{I} 2=0 \%$ ) or improve intracranial pressure control (weighted mean difference $-1.25 \mathrm{~mm} \mathrm{Hg}, 95 \% \mathrm{CI}-4.18$ to $1.68, \mathrm{I} 2=78 \%$ ) as compared to any other solutions. Only one study reported monitoring for adverse events with hypertonic saline, finding no significant differences between comparison groups.

Conclusions We observed no mortality benefit or effect on the control of intracranial pressure with the use of hypertonic saline when compared to other solutions. Based on the current level of evidence pertaining to mortality or control of intracranial pressure, hypertonic saline could thus not be recommended as a first-line agent for managing patients with severe traumatic brain injury.

\section{Emergencias}

Official Journal of the Spanish Society of Emergency Medicine

Unmanned aerial vehicles: usefulness for victim searches and triage in disasters

Pardo Ríos M, Pérez Alonso N, Lasheras Velasco J, Juguera Rodríguez L, López Ayuso B, Muñoz Solera R, Martínez Riquelme C, Nieto Fernández-Pacheco A.

Objective To analyze the influence of drones equipped with thermal cameras for finding victims and aiding triage during disasters.

Methods We carried out a prospective, crosssectional analysis and 6 experimental simulations, each with 25 victims to locate and triage. Nurses were randomized to a control group or a drone group. Drone-group nurses were given access to

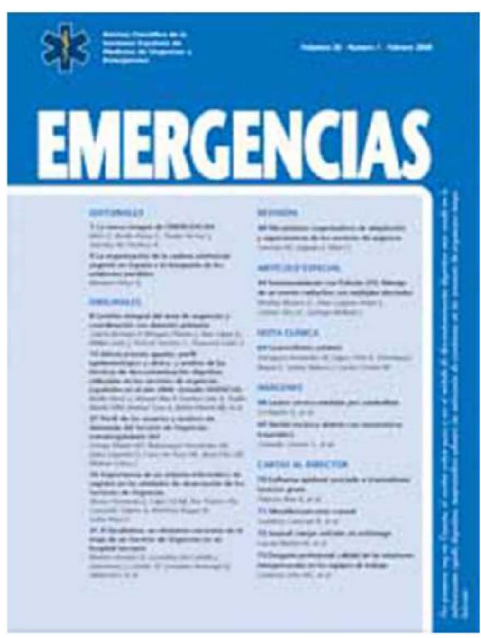
images from the thermal cameras 10 minutes before the exercise started.

Results The mean (SD) distance the nurses searched in the control group (1091.11 [146.41] m) was significantly greater than the distance searched by nurses in the drone group ( 920 [71.93] $\mathrm{m}(\mathrm{p}=0.0031)$. The control group found a mean of $66.7 \%$ of the victims, a significantly smaller percentage than the drone group's mean of $92 \% \quad(p=0.0001)$. Triage quality (undertriage and overtriage) was similar in the 2 groups as shown by maneuvers undertaken to open airways and control bleeding.

Conclusion Drones with thermal cameras were useful in searching for victims of simulated disasters in this study, although they had no impact on the quality of the nurses' triage. 